\title{
Possible CASE OF HUMAN BRUCELLOSIS
}

Laurie C Pryde, Senior Field Veterinary Officer, Dubbo John Hall, Formerly Director, Western New South Wales Public Health Unit

Ed Kraa, Senior Policy Advisor, Infectious Diseases Section

A 49-year-old male from the Dubbo district became ill on a trip to Queensland and was admitted to hospital with pyrexia of unknown origin on January 17, 1993. Symptoms included a high fever, rigors, a nervous twitch and night sweats.

\section{HISTORY}

Between January 5 and 7,1993 , the patient assisted with the slaughter and butchering of an 18-month-old steer on a property in the Coonabarabran Rural Lands Protection District. The property had just been sold and all remaining livestock was being disposed of. Neither of the other two people assisting in the slaughtering and butchering displayed any ill-health since early January.

The herd testing data obtained on tail tag numbers showed the herd of origin of the slaughtered steer had been certified brucellosis-free since 1984. Abattoir monitoring of seven cattle from the property between October 1990 and June 1992 had also been negative for brucellosis.

The patient maintained that until his involvement with the slaughtering of the steer he had had no association with cattle for the past 20 years nor had he previously suffered an illness similar to that he experienced in January. As a young man he worked on a station near Armidale that was confirmed to have brucellosis.

\section{CLINICAL COURSE}

The patient remained in hospital for three weeks, during which he was clinically diagnosed as having Ross River fever. The diagnosis was presumably made on the basis of clinical signs and initial positive serology but repeat serology returned a negative result and the first result was later considered to be a false positive. When discharged, no definitive diagnosis had been established. Only after discharge did the results of other serology tests which were positive for brucella become available.

Discharge papers sent to the patient's medical practitioner suggested it may be worthwhile to perform repeat serology for brucellosis and cytomegalovirus. Subsequent serology appeared to confirm a diagnosis of brucellosis infection. The three sera were retested at the Institute of Clinical Pathology and Medical Research at Westmead Hospital in April. Results are shown in Table 4.

\begin{tabular}{|c|c|c|}
\hline \multicolumn{3}{|c|}{$\begin{array}{l}\text { BRUCELLA SEROLOGY } \\
\text { TESTING RESULTS } \\
\text { APRIL } 1993\end{array}$} \\
\hline Date & $\begin{array}{c}\text { Standard Agglutination } \\
\text { Test (SAT) }\end{array}$ & AHG (Coombs) Test \\
\hline 17.1 .93 & Titre $<20$ & 80 \\
\hline 1.2 .93 & Titre 160 & 320 \\
\hline 22.3 .93 & Titre $<160$ & 320 \\
\hline
\end{tabular}

\section{DISCUSSION}

The infectious agents of brucellosis in humans are Brucella abortus, B. melitensis, B. suis and B. canis of which two$B$. abortus and B. suis - are endemic in Australia ${ }^{1}$.
B. abortus affects cattle and other boviae. B. suis usually affects pigs but can be transmitted to cattle ${ }^{2}$. Human brucellosis occurs most commonly in people such as farmers, veterinarians and abattoir workers, who are occupationally exposed to infected domestic animals, or in people who drink unpasteurised cows' milk. Brucellosis is a systemic disease with acute or insidious onset, characterised by continued, intermittent or irregular fever of variable duration, headache, weakness, profuse sweating, chills, arthralgia, depression, weight loss and generalised aching. Localised suppurative infections may occur. Infections are frequently subclinical and unrecognised. Onset is variable, commonly ranging from $5-60$ days but can be as long as several months. Duration can also vary, ranging from several days to occasionally a year or more ${ }^{3}$. Blood cultures are recommended for unequivocal diagnosis of brucellosis.

A national brucella and tuberculosis eradication campaign was begun in the early $1970 \mathrm{~s}$. The campaign targeted bovine $B$. abortus with a number of control strategies. A vaccination program initially targeted at heifer calves, followed by a vaccination program for adult cattle, was undertaken. Blood samples were collected at abattoirs for testing and, where positive, tail tag tracing was undertaken, followed by herd testing on implicated properties. Bulk milk samples from dairy farms were also tested and implicated properties were similarly herd tested. Properties that came to notice because of abortion problems were also followed up with herd testing. Subsequently a program of testing every cattle herd in NSW was undertaken. Herds which had no history of brucellosis were tested at least three times. Herds with problems were followed up at two-month intervals. Compensation was paid to farmers for stock that was destroyed.

The program was effective and Australia was declared free of bovine brucellosis in July 1989. Human brucellosis declined concomitantly until the mid-1980s, when an increase was observed in Central and South-East Queensland - predominantly due to $B$. suis associated with feral pig hunting and processing.

Blood cultures were not performed in this case and it is not possible to distinguish $B$. abortus from $B$. suis infection on serology alone. There was no history of exposure to feral pigs during the travel to Queensland and it is unlikely that this was a case of $B$. suis infection following recent exposure to feral pigs. It is possible the serology reactions were due to a very old infection in the light of the patient's contact with B. abortus-infected cattle on the Armidale property some 20 years previously. The incubation period seems very short if the source of infection was the animal slaughtered 10 days before the onset of illness, however this is not impossible. As the man travelled to Queensland after the slaughtering of the steer the incubation period for any $B$. suis infection acquired in Queensland would be even shorter.

Investigation of human cases should try to identify any animal contacts in the three months before onset. Isolation by blood culture should be attempted in suspected cases of human brucellosis as it is of importance to identify the infective organism to assist in determining the source and the possibility of $B$. abortus infection in the domestic animal population.

1. Robson JM, Harrison MW. Wood RN, Tilse MH, McKay AB and Brodribb TR. Brucellosis: re-emeruence and changing epidemiolog in Queensland. Medical doumal of Australia 1993;159:153-158. 2 Gilbert GL. Brucellosis: cont inuinr risk. Med J Aust 1993-159:147-148. 3. Benenson AS. Control of ('mmmunicable Diseases in Man. 15th edition, Washington: American I'ublic Health Association, 1990. 